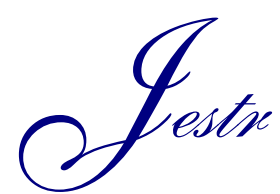

Research Article

\title{
Connectivity Reliability Analysis of Road Network of Multiple OD Pairs based on the Structural Reliability of Joint Failure Modes
}

\author{
Bai Shi-ye* and Zhu Jie-jiang \\ Department of Civil Engineering, Shanghai University, Shanghai, 200072, China
}

Received 28 March 2016; Accepted 15 December 2016

\begin{abstract}
In order to improve operational efficiency of traffic road, it is necessary to carry out the exploration on the reliability of road network. The connectivity reliability is the critical index to calculate the reliability of the road network. Based on the joint failure modes of fatigue cracking and permanent deformation, the connectivity reliability model of road network was established, which was further introduced into the reliability analysis of road network system. By considering the influences of different OD (Origin-Destination) pairs and their weighting coefficients on the system reliability, the reliability model of complex road network of multiple OD pairs was finally constructed. Furthermore, connectivity reliability of complex road network was calculated on the basis of the Monte Carlo method and the breadth-first search method. The influences of the different road elements and nodes on the system reliability were analyzed by the numerical examples. In addition, the influences of reinforcement and expansion of the road network on the system reliability were also evaluated. Results show that, although the presented models based on multiple OD pairs and its different weighing coefficients are closer to the actual road network system, it is safer to define the road network reliability by single OD pair. Moreover, the failure of the nodes, especially those adjacent to the starting node, causes more damages to the road network system than the road section does, which decrease the reliability of the road network system. The recovery of the road sections adjacent to the starting nodes can not only effectively improve the reliability of the system but also reduce economic costs.
\end{abstract}

Keywords: Joint Failure Modes; Weighting Coefficient of Multiple OD Pairs; Connectivity Reliability of Road Network; Reinforcement and Expansion of Road Network

\section{Introduction}

With the rapid development of construction of urban road, the congestion problem of urban road traffic has attracted more attentions. Thus, the analysis on connectivity reliability model of road network plays a key role in dealing with this problem. Presently, the scope of studies on the traffic network reliability mainly includes three aspects, namely connectivity reliability [1-3], travel time reliability [4-6] and capacity reliability [7-9]. The connectivity reliability was the earliest research field among the research on the road network reliability. It began to attract more concerns after the earthquake in Kobe, Japan. After multiple 8-magnitude earthquakes took place in China, the research on connectivity reliability became one of the most popular research field [10-15]. Only when the road network remains connective, the normal travel can be guaranteed. Therefore, the connectivity reliability is the critical index to calculate the reliability of the road network.

\section{State of the Art}

The connectivity reliability was firstly proposed by Mine and Kawai in 1982 [16]. They defined it as the probability of the connectivity between two points in the road network. In

\footnotetext{
- E-mail address: bai880602@sina.com

ISSN: $1791-2377$ C 2016 Eastern Macedonia and Thrace Institute of Technology. All rights reserved.
}

1989, Iida and Wakabayashi studied the connectivity reliability of the special OD pairs based on the minimal path set algorithm and cut set algorithm [17],which extended the connectivity reliability of two points in road network to the whole network. Bell et al. adopted the graph theory to calculate the connectivity reliability [18]. In their study, only two running states (complete connection and complete interruption) were considered, and the importance of the road section was ignored. In 1996, Hu presented a new method to compute reliability of disjointed network [19]. In 2005, Stephen and David proposed a way to estimate the probability distribution of total network travel time [20].In 2008, Zhang et al. initially presented the network capacity reliability index based on topological structure [21]. In 2009, Erik investigated the way in which the geographical disparities depended on the road network structure and travel patterns. He proposed aggregate supply-side as well as demand-side indicators, and combined them in statistical regression models [22]. In 2014, Kenetsu developed two models which simultaneously estimated the value of travel time and its reliability based on the route choice behavior of risk-averse driver [23]. In 2015, Chong et al. presented a bilevel programming model based on the unblocked reliability of urban network [24].

Among the previous studies on connectivity reliability of the whole network, the road structural reliability and network reliability were treated as two main research subjects that were independent from each other from the 
perspective of macro management. However, there is an inevitable link between these two subjects. The road structural reliability is the foundation of researching the road network reliability. The change of the road structural reliability must affect the pavement bearing capacity, which can determine the road bearing traffic flow. The change of the traffic flow of road section will influence the traffic flow of the adjacent road. At the same time, the whole road network reliability will also undergo changes. Therefore, it is essential to explore the reliability of road network on the basis of the road structure reliability. In addition, graph approaches are usually used in the traditional connectivity reliability calculation. This kind of method is with high accuracy and can be applied into the connectivity reliability calculation of low density and small-scale road network. Nevertheless, it is not applicable to the large-scale and complicated traffic network because the calculation amount is too huge and it is difficult to reflect the real situation of the road network. The traditional calculation of connectivity reliability of road network is mostly based on single OD pairs. Even if multiple OD pairs are introduced into the reliability calculation of road network, the model can't distinguish the different effects between different OD pairs. In fact, different OD pairs have a different influence on the road network performance and damage probability. Thus, by considering the importance of different OD pairs, the road network reliability evaluation can be better complied with the actual situation.

The remainder of this study was organized as follows. Section 3 established the reliability model based on the joint failure modes of fatigue cracking and permanent deformation, and calculated the reliability of asphalt pavement structure. By introducing the structural reliability into the road network connectivity analysis, the connectivity reliability model of road network was constructed based on the complex multiple OD pairs and weighting coefficients. Furthermore, the Mote Carlo method and the bread-first search method were utilized to obtain the connectivity reliability of the complex road network system. Section 4 analyzed the influence of the road section and nodes failure on the connectivity reliability of road network system through numerical examples. Moreover, this section discussed the effect of reinforcement and expansion of the road network on the reliability recovery of road network. Finally, Section 5 summarized the conclusions.

\section{Methodology}

\subsection{Reliability of Asphalt Pavement Structure Under Multiple Failure Modes}

\subsubsection{Fatigue cracking failure mode}

The transfer functions of fatigue cracking failure mode are generally utilized to calculate the number of load repetitions that lead to the failure of the pavement. In this study, the transfer function of fatigue cracking is taken as the following form:

$$
N_{f c}=c_{1} \cdot \varepsilon_{t}^{-c_{2}} \cdot E_{1}^{-c_{3}}
$$

where $N_{f c}$ is the cumulative number of standard axle load repetition that produces $20 \%$ cracking area; $\varepsilon_{t}$ is the tensile strain at the bottom of asphalt surface layer; $E_{1}$ is the elastic modulus of asphalt surface layer; $c_{1}, c_{2}$, and $c_{3}$ are regression coefficients of the fatigue cracking equation.

\subsubsection{Rutting failure mode}

The relationship between the cumulative number of standard axle load repetition that leads to permanent rutting deformation and the vertical compressive strain at the top of the subgrade is described as the following transfer function:

$$
N_{r}=c_{4} \cdot \varepsilon_{c}^{c_{5}}
$$

Where $N_{r}$ is the cumulative number of standard axle load repetition that produces $12.7 \mathrm{~mm}$ rutting; $\varepsilon_{c}$ is the vertical compressive strain at the top of the subgrade; $c_{4}$ and $c_{5}$ are regression coefficients of the rutting equation.

\subsubsection{Reliability of asphalt pavement structure}

As for most of the practical engineering structures, there are actually multiple failure modes. Thus, the reliability of the asphalt pavement should be defined as the system reliability. For the asphalt pavement structure, the system will fail if either fatigue cracking or rutting occurs. The failure probability of two failure modes of the pavement structure can be expressed as follows:

$$
p_{f}=\mathrm{P}\left(\underset{i=1}{\mathrm{U}} Z_{i} \leq 0\right)=\int_{\bigcup_{i=1}^{2} Z_{i} \leq 0} f_{X}(x) d x
$$

where $Z_{i}$ is the functional equation ( $\mathrm{i}=1,2$ represents fatigue cracking and rutting event respectively). Its function is given as follows:

$$
Z_{1}(x)=N_{f c}-N_{d} ; Z_{2}(x)=N_{r}-N_{d}
$$

where $N_{d}$ is the limiting number of traffic cycling load repetition.

The Monte Carlo Method is widely applied in reliability analysis of engineering structures. Function $M(x)$ is utilized to denote the failure state $(M(x)=1)$ or the safe state $(M(x)=0)$, and $x$ is random variable that depends on parameters such as material, load, and structure size. Based on generation algorithm, a sequence of input variable $x_{i}$ is obtained according to the joint statistical distribution. Then the failure probability of the asphalt pavement structure can be estimated by the following formulation:

$$
p_{f}=\frac{\sum_{i=1}^{N_{s}} M\left(x_{i}\right)}{N_{s}}
$$

where $N_{s}$ is the total simulation number of Monte Carlo Method.

Firstly, fatigue cracking and rutting are determined respectively by the tensile strain $\varepsilon_{t}$ at the bottom of the asphalt surface layer and the vertical compressive strain $\varepsilon_{c}$ at the top of the subgrade. The pavement design general software KENPAVE is utilized to predict the critical strain 
$\varepsilon_{t}$ and $\varepsilon_{c}$. Secondly, according to the equations of (1) and (2), the number of load repetitions that produce fatigue cracking and rutting respectively is obtained. Finally, the failure probability function (3) is solved by Monte Carlo Method. Furthermore, the joint failure probability based on two failure modes and the reliability of asphalt pavement structure are obtained.

\subsection{Road Connectivity Reliability of Complex Road Network with Multiple OD Pairs}

\subsubsection{Reliability of road section}

Based on the above failure modes, the reliability of the road section is described as follows:

$R_{i j k}=P\left(N_{f c}<N_{d}, N_{r}<N_{d}\right)$

where $R_{i j k}$ is the reliability of the $k$-th road section of the $j$-th path of the $i$-th OD pair.

\subsubsection{Reliability of path}

The path is a route from the starting point of any OD pair to the ending point. It is composed of multiple road sections in series. Thus, the path reliability can be defined as:

$R_{i j}=\prod_{k=1}^{m} R_{i j k}$

where $R_{i j}$ is the reliability of the $\mathrm{j}$-th path of the $\mathrm{i}$-th OD pair.

\subsubsection{OD pair reliability}

The OD pair is regarded as multiple paths in parallel, shown as follows:

$$
R_{i}=1-\prod_{j=1}^{n}\left(1-R_{i j}\right)
$$

where $R_{i}$ is the reliability of the $i$-th OD pair.

\subsubsection{The traditionally defined reliability of road network}

The reliability of road network is expressed as follows:

$R=\frac{\sum R_{i}}{n}$

where $R$ is the traditionally defined reliability of the road network.

\subsubsection{The improved reliability model with multiple OD} pairs and its weighing coefficients

Previous calculation method of the road network reliability is based on the average reliability of all the OD pairs. However, the relative importance of different road sections and nodes haven't been considered. Therefore, the road network reliability model should be improved as follows:

$P_{n e t}=\frac{\sum_{i=1}^{n}\left[w(i) \times P_{O D}(i)\right]}{\sum_{i=1}^{n} w(i)}$ where $P_{n e t}$ is the reliability of road networks based on the presented model, $P_{O D}(i)$ is the reliability of the i-th OD pair, $w(i)$ is weighting coefficients of the $i$-th OD pair, and $n$ is the number of all OD pairs. When the number of nodes is $m$, $n=\frac{m(m-1)}{2}$.

The weighting coefficient of the $i$-th OD pair is expressed as follows:

$$
w(i)=\alpha_{1} \times \frac{P_{i}}{\bar{P}}+\alpha_{2} \times \frac{G_{i}}{\bar{G}}
$$

where $\alpha_{1}$ and $\alpha_{2}$ are weighting coefficients of population numbers and GDP respectively, $\alpha_{1}+\alpha_{2}=1, P_{i}$ is the population of the $i$-th OD pair, $G_{i}$ is the GDP of the i-th OD pair, $\bar{P}$ is the population of all OD pairs, and $\bar{G}$ is the GPD of all OD pairs.

3.2.6 The calculation of the connectivity reliability of road network

Based on the Monte Carlo method, the connectivity reliability of road network is calculated through MATLAB programming. The implementation procedure is shown in Figure 1.

Firstly, some parameters are predefined including road section numbers $\mathrm{r}$, reliability matrix of road section $\mathrm{a}(i, j)(i$ and $\mathrm{j}$ are the nodes of the road section), connectivity coefficient matrix $\mathrm{A}(i, j)(\mathrm{A}(i, j)=1$ when the road section with nodes $i$ and $j$ is connective, or $\mathrm{A}(i, j)=0)$, and failure information matrix of road section $\mathrm{c}\left(\mathrm{nS}, n^{2}\right)(\mathrm{nS}$ denotes simulation number, and $\mathrm{n}$ signifies node number.) Secondly, the demand connectivity reliability of road sections is postulated to obey the uniform distribution in the interval of $[0,1]$. When the demand connectivity reliability of road section is lower than the connectivity reliability, then the road sections are linked, which means $\mathrm{A}(i, j)=1$, or $\mathrm{A}(i, j)=0$. Besides, the failure information matrix of road section $\mathrm{c}$ is assigned. Thirdly, the road section of the network is searched by breadth-first-search method, in order to judge the connectivity of the OD pair. If connective, the cumulative time NF increases by one time, or it will not increase. Loop computation is performed to acquire satisfying simulation numbers. Finally, the connectivity reliability of road network can be obtained.

\section{Result Analysis and Discussion}

\subsection{Example}

As shown in Figure 2, a certain area of the road network in Shanghai is selected as the computational example. The improved reliability model is chosen to calculate the reliability of the road network.

According to the above road network system, some main road sections are chosen to be analyzed and marked in red, as shown in Figure 3. Finally, the simplified road network system is obtained and presented in Figure 4.

There are 15 nodes $(\mathrm{N} 1 \sim \mathrm{N} 15)$ in the road network system, including 22 road sections (R1 R22), and 9 OD pairs $(\mathrm{N} 1 \rightarrow \mathrm{N} 7, \mathrm{~N} 1 \rightarrow \mathrm{N} 11, \mathrm{~N} 1 \rightarrow \mathrm{N} 15, \mathrm{~N} 2 \rightarrow \mathrm{N} 7, \mathrm{~N} 2 \rightarrow \mathrm{N} 11$, $\mathrm{N} 2 \rightarrow \mathrm{N} 15, \mathrm{~N} 3 \rightarrow \mathrm{N} 7, \mathrm{~N} 3 \rightarrow \mathrm{N} 11$, and N3 $\rightarrow \mathrm{N} 15)$. 


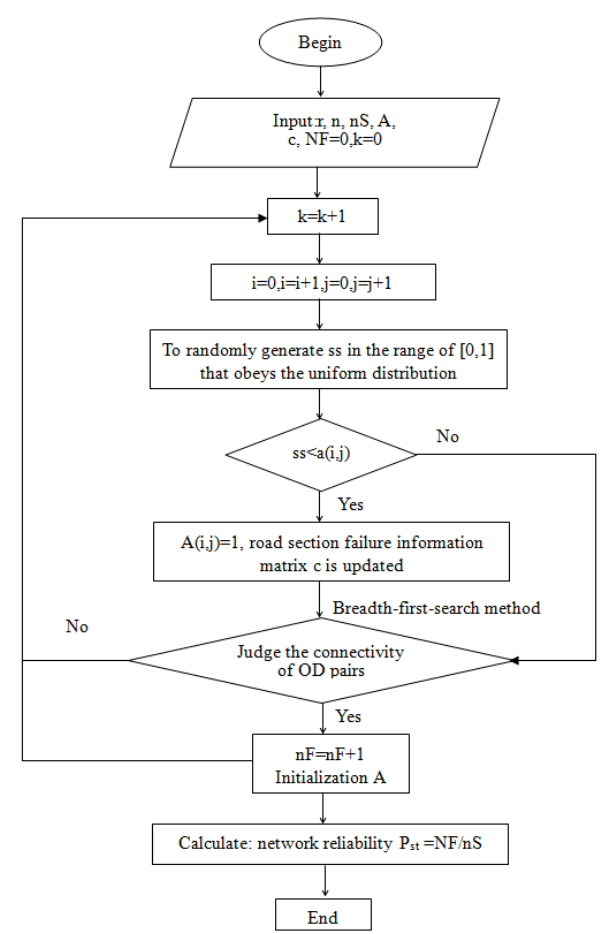

Fig. 1. The calculation procedure of the road network connectivity reliability

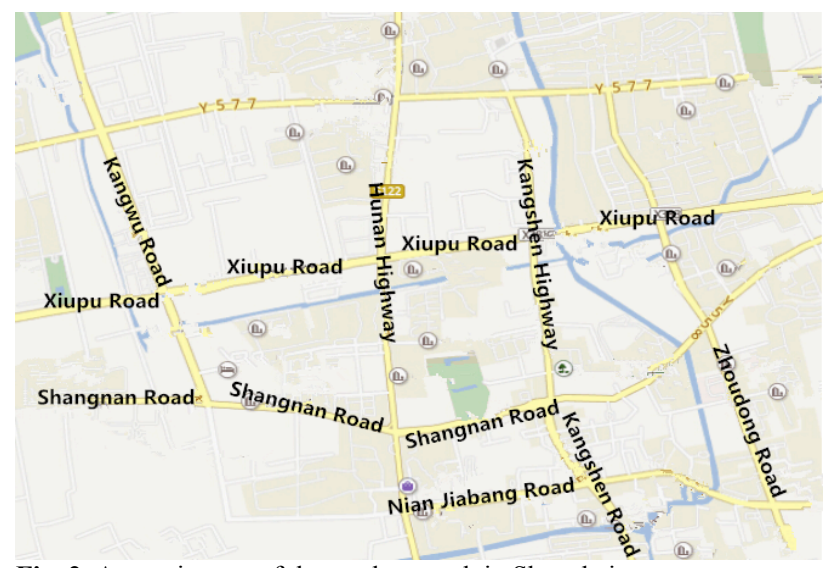

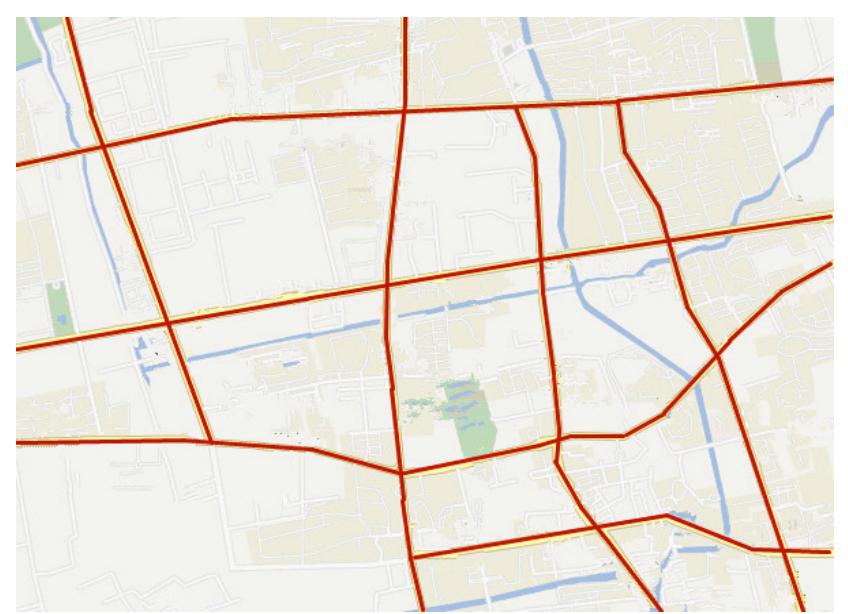

Fig. 3. The sketch graph of road network

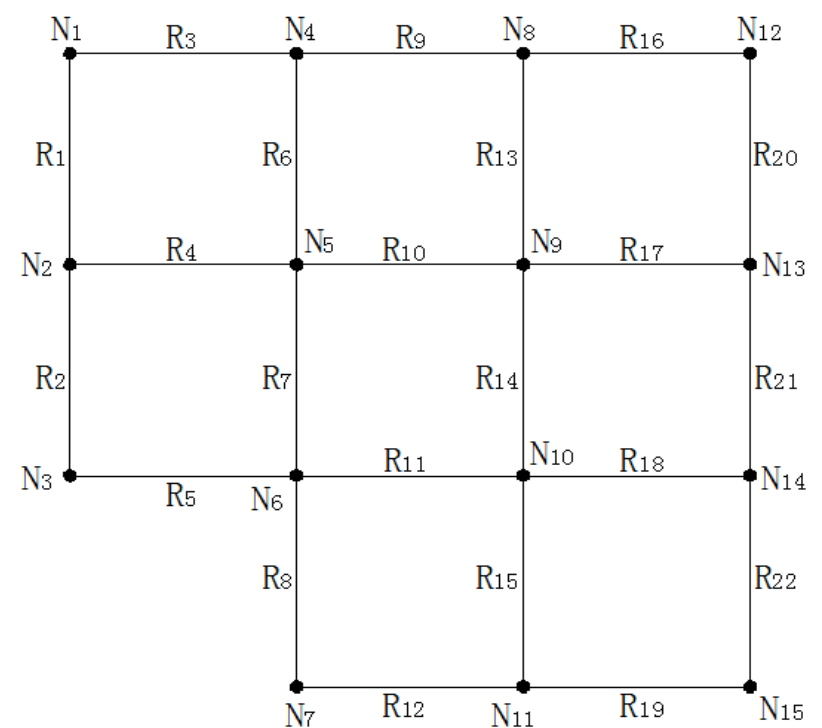

Fig. 4. The simplified road network system

The road section reliability is based on the joint failure modes of structure. The weighting coefficients of population and GPD are both 0.5 . All the road sections reliability and path parameters are shown in Table 1 and Table 2 respectively.

Fig. 2. A certain area of the road network in Shanghai

Table 1. The road section reliabilities in the network system

\begin{tabular}{c|c|c|c|c|c|c|c|c|c|c|c}
\hline Road section number & $R_{1}$ & $R_{2}$ & $R_{3}$ & $R_{4}$ & $R_{5}$ & $R_{6}$ & $R_{7}$ & $R_{8}$ & $R_{9}$ & $R_{10}$ & $R_{11}$ \\
\hline Reliability & 0.76 & 0.80 & 0.72 & 0.83 & 0.93 & 0.81 & 0.86 & 0.71 & 0.84 & 0.73 & 0.91 \\
Path number & $R_{12}$ & $R_{13}$ & $R_{14}$ & $R_{15}$ & $R_{16}$ & $R_{17}$ & $R_{18}$ & $R_{19}$ & $R_{20}$ & $R_{21}$ & $R_{22}$ \\
reliability & 0.87 & 0.72 & 0.81 & 0.77 & 0.85 & 0.75 & 0.79 & 0.88 & 0.90 & 0.91 & 0.81 \\
\hline
\end{tabular}

Table 2. Parameters of all road sections in the network system

\begin{tabular}{c|c|c|c|c|c|c|c|c|c|c|c}
\hline Road section number & $R_{1}$ & $R_{2}$ & $R_{3}$ & $R_{4}$ & $R_{5}$ & $R_{6}$ & $R_{7}$ & $R_{8}$ & $R_{9}$ & $R_{10}$ & $R_{11}$ \\
\hline$P_{i} / 10^{4}$ & 0.075 & 0.048 & 0.056 & 0.033 & 0.074 & 0.078 & 0.043 & 0.038 & 0.051 & 0.065 & 0.075 \\
$G_{i} / 10^{4}$ & 820 & 140 & 550 & 100 & 570 & 700 & 650 & 600 & 480 & 560 & 250 \\
Path number & $R_{12}$ & $R_{13}$ & $R_{14}$ & $R_{15}$ & $R_{16}$ & $R_{17}$ & $R_{18}$ & $R_{19}$ & $R_{20}$ & $R_{21}$ & $R_{22}$ \\
$P_{i} / 10^{4}$ & 0.045 & 0.068 & 0.051 & 0.023 & 0.078 & 0.068 & 0.047 & 0.018 & 0.031 & 0.045 & 0.055 \\
$G_{i} / 10^{4}$ & 620 & 240 & 350 & 130 & 670 & 400 & 350 & 200 & 580 & 660 & 350 \\
\hline
\end{tabular}

In this study, the breadth-first-search method is combined with the Monte Carlo method, which is used to calculate the reliability of all OD pairs in the road network system. The simulation number is one hundred thousand. The reliability results of all OD pairs and the weighting coefficients are listed in Table 3. 
Table 3. The reliabilities of all OD pairs in road network system

\begin{tabular}{c|c|c|c|c|c|c|c|c|c|c}
\hline \multirow{2}{*}{ OD pair } & Start & $N_{1}$ & $N_{1}$ & $N_{1}$ & $N_{2}$ & $N_{2}$ & $N_{2}$ & $N_{3}$ & $N_{3}$ & $N_{3}$ \\
\cline { 2 - 23 } & \multirow{2}{*}{ End } & $N_{7}$ & $N_{11}$ & $N_{15}$ & $N_{7}$ & $N_{11}$ & $N_{15}$ & $N_{7}$ & $N_{11}$ & $N_{15}$ \\
\hline \multicolumn{2}{c}{ Reliability } \\
Weighting coefficient & 0.8581 & 0.8837 & 0.8717 & 0.9225 & 0.9444 & 0.9338 & 0.9240 & 0.9493 & 0.9311 \\
0.1393 & 0.1660 \\
\hline
\end{tabular}

As shown in Table 3, the minimum reliability of all OD pair is 0.86 and the maximum is 0.95 . The weighting coefficients of different OD pairs are different, so that the relative contributions of different OD pairs are also diverse. Based on Equation (10), the reliability of road network system is calculated as 0.9067 . When this value is compared with the different single OD pair results shown in Table 3, it can be found that the accurate system reliability cannot be evaluated by using a single OD pair. For example, the system reliability based on the OD pair N3 $\rightarrow$ N11 is 0.9493 , whereas the reliability based on multiple OD pairs model is 0.9067 . The maximum error between them reaches $4.26 \%$, and it is not safe. Therefore, the improved evaluation model in this study is closer to the real engineering situation, which can lay a good foundation for the computation and analysis of the reinforcement and expansion of more complex road network systems.

\subsection{The Influence of Failure and Reinforcement of Road on System Reliability}

\subsubsection{Road section failure}

On the basis of the above example results, the influence of the failure of road section and nodes on system reliability is further analyzed. In this study, only one OD pair (N1 N15) is selected, while all the other parameters remain unchanged. The simulation time is one hundred thousand under the Monte Carlo method.

There are three forms of road section failures, namely the adjacent road section failure, the second adjacent road section failure and non-adjacent road section failure. Three cases are chosen to be analyzed, and their system reliabilities are respectively calculated and shown in Table 4- 6.

Table 4. The road network reliability of adjacent road sections to $N_{1}$ and $N_{15}$

\begin{tabular}{c|c|c|c|c}
\hline Failure road section & $R_{1}$ & $R_{3}$ & $R_{19}$ & $R_{22}$ \\
\hline Reliability & 0.6501 & 0.6946 & 0.7148 & 0.7209 \\
\hline
\end{tabular}

Tab.5. The road network reliability of secondly adjacent road sections to $N_{1}$ and $N_{15}$

\begin{tabular}{c|c|c|c|c}
\hline Failure road section & $R_{2}$ & $R_{4}$ & $R_{6}$ & $R_{9}$ \\
\hline Reliability & 0.8332 & 0.8253 & 0.8356 & 0.8258 \\
Failure road section & $R_{12}$ & $R_{15}$ & $R_{18}$ & $R_{21}$ \\
Reliability & 0.8387 & 0.8151 & 0.8525 & 0.8328 \\
\hline
\end{tabular}

Table 6. The road network reliability of non-adjacent road sections to $N_{1}$ and $N_{15}$

\begin{tabular}{|c|c|c|c|c|c|}
\hline Failure road section & $R_{5}$ & $R_{7}$ & $R_{8}$ & $R_{10}$ & $R_{11}$ \\
\hline Reliability & 0.8332 & 0.8478 & 0.8417 & 0.8528 & 0.8414 \\
\hline Failure road section & $R_{13}$ & $R_{14}$ & $R_{16}$ & $R_{17}$ & $R_{20}$ \\
\hline Reliability & 0.8617 & 0.8564 & 0.8582 & 0.8642 & 0.8489 \\
\hline
\end{tabular}

From Table 4-6, it can be seen that the road network system reliability can decline by as much as $22 \%$ compared with the previous complete road network system, when the road sections adjacent to the starting and ending points fail. It decreases by as much as $6 \%$ when the secondary adjacent road sections fail, and only $4 \%$ when the non-adjacent road sections fail. By comparing the above three cases with each other, it can be found that the road sections adjacent to the starting and ending points influence the system reliability most significantly, followed by the secondary adjacent road sections. That is to say, the non-adjacent road sections' influence on is the least prominent, which suggests that the road network system is stable.

\subsubsection{Road node failure}

There are two forms of road node failure, namely the adjacent and non-adjacent road node failures. Two cases are chosen to be analyzed, and their system reliabilities are calculated and shown in Table 7 and Table 8.

Tab.7. The road network reliability of adjacent road nodes to $N_{1}$ and $N_{15}$

\begin{tabular}{c|c|c|c|c}
\hline Failure road node & $N_{2}$ & $N_{4}$ & $N_{11}$ & $N_{14}$ \\
\hline Reliability & 0.6495 & 0.6768 & 0.7173 & 0.7156 \\
\hline
\end{tabular}

Table 8. The road network reliability of non-adjacent road nodes to $N_{1}$ and $N_{15}$

\begin{tabular}{c|c|c|c|c|c}
\hline $\begin{array}{c}\text { Failure road } \\
\text { node }\end{array}$ & $N_{3}$ & $N_{5}$ & $N_{6}$ & $N_{7}$ & $N_{8}$ \\
\hline $\begin{array}{c}\text { Reliability } \\
\text { Failure road } \\
\text { node }\end{array}$ & 0.8397 & 0.7665 & 0.7416 & 0.8423 & 0.8222 \\
$\begin{array}{c}\text { Failure road } \\
\text { node }\end{array}$ & 0.8322 & $N_{10}$ & $N_{12}$ & $N_{13}$ & \\
\hline
\end{tabular}

From Table 7 and Table 8, it can be seen that the road network system reliability declines by as much as $23 \%$ compared with the previous complete road network system when the road nodes adjacent to the starting and ending points fail. It decreases by $12 \%$ when the non-adjacent road nodes fail. In accordance to the result of comparison between the above two cases, the road nodes adjacent to the starting and ending points more influence the system reliability (as shown in Tables 4-6). In addition, the nonadjacent node failure influences the system reliability more than the non-adjacent road section failure does. Thus, it is more important to ensure the safety and reliability of road nodes rather than road sections for the same road network system.

\subsubsection{Road reinforcement and expansion}

In order to further discuss the influence of the reinforcement and expansion of road on the road network system reliability, the case of $\mathrm{N} 2$ failure is analyzed. The other parameters are the same as those in the previous example.

When the nodes fail, there are two approaches to recover the reliability of the road network system, namely (1) Reinforcement of the other road sections, (2) Expansion of 
the road network. In our subsequent studies, the influence of the two approaches on the reliability of road system will be discussed.

As N2 fails, the other road sections adopt the same form of reinforcement, and then the reliability of reinforced road sections will be enhanced. The reinforcement forms are given in Table 9.

Table 9. Reliability after reinforcement

\begin{tabular}{c|c|c|c|c|c}
\hline $\begin{array}{c}\text { Reinforcement level } \\
\text { of other road } \\
\text { sections }\end{array}$ & $\mathbf{0 \%}$ & $\mathbf{5 \%}$ & $\mathbf{1 0 \%}$ & $\mathbf{1 5 \%}$ & $\mathbf{2 0 \%}$ \\
\hline $\begin{array}{c}\text { Reliability } \\
\text { Reinforcement level } \\
\text { of other road } \\
\text { sections } \\
\text { Reliability }\end{array}$ & 0.6495 & 0.7167 & 0.7743 & 0.8264 & 0.8617 \\
\hline
\end{tabular}

Obviously, the system reliability increases nonlinearly with the improvement of the road reinforcement level. When the reinforcement degree increases from 0 to $10 \%$, the reliability grows from 0.6495 to 0.7743 (rising by $14 \%$ ). When the reinforcement degree increases from $10 \%$ to $20 \%$, the reliability grows from 0.7743 to 0.8617 (rising by $9 \%$ ). As the reinforcement degree continues to grow to $40 \%$, the reliability reaches $100 \%$. Consequently, it is an effective way to improve the system reliability by reinforcing the nonfailure road sections.

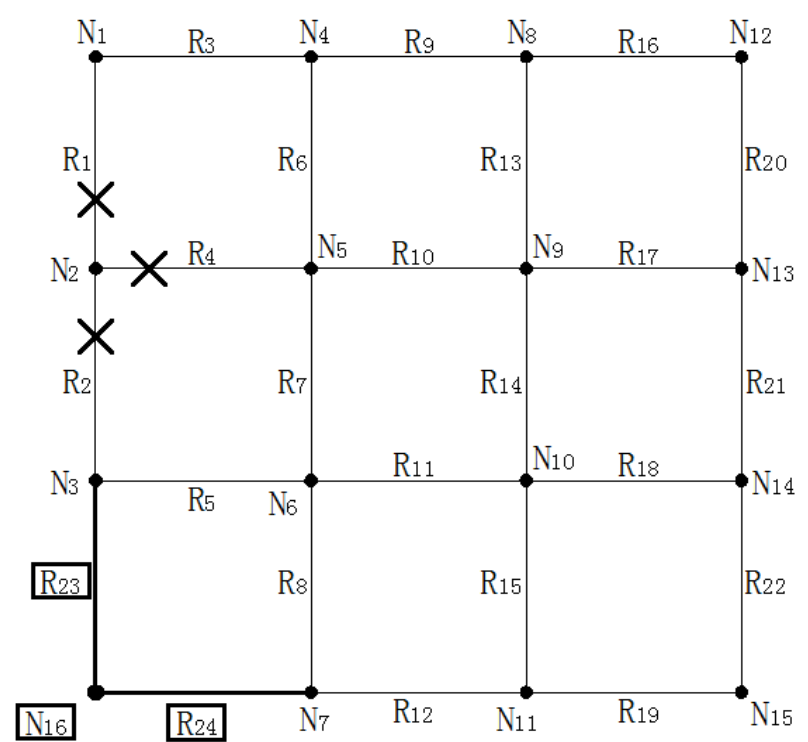

Fig.5. The expansion of network--Approach 1

When the node $\mathrm{N} 2$ fails, the expansion of the network can be used to recover the reliability of the road system. Figures 5-7 display three forms of expansion.

As depicted in Figure 5, the failure of N2 will lead to the failure of road sections R1, R2 and R4. At this time, the reliability of the road network system is 0.6495 . By adding the node N16 and road section R23 and R24, the reliability can be recovered. The initial condition $(\mathrm{P}(\mathrm{R} 23)=0.81$, $\mathrm{P}(\mathrm{R} 24)=0.88)$ is set. Then, the road network system reliability is calculated as 0.6516 based on Approach 1 . Compared with the previous road network system, it is not effective to improve the reliability of road network system with this approach.

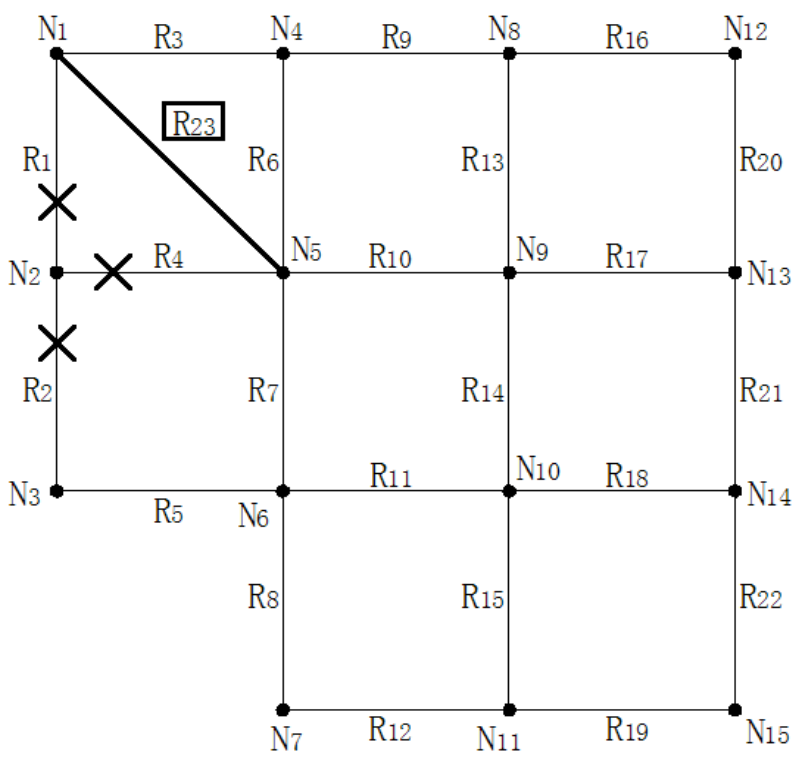

Fig. 6. The expansion of network--Approach 2

As shown in Figure 6, R23 is added, and the initial condition $P(R 23)=0.78$ is set, after the failure of $N 2$. Then, the reliability of the road network system is calculated as 0.9358 based on Approach 2. Compared with the previous system, the reliability has a notable increase.

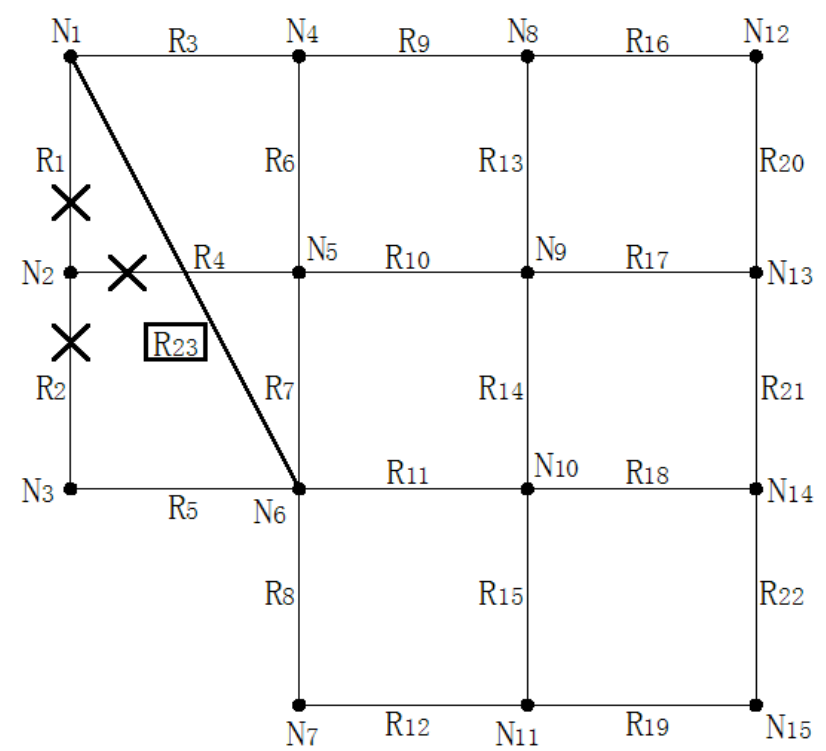

Fig. 7. The expansion of network--Approach 3

As shown in Figure 7, R23 is added, and the initial condition $\mathrm{P}(\mathrm{R} 23)=0.78$ is set after the failure of $\mathrm{N} 2$. Then, the reliability of the road network system is calculated as 0.9368 based on Approach 3. Compared with the previous system, the reliability also has a remarkable increase.

In the light of the comparison results among the three approaches of expanding the road network, although one node and two road sections are added into the system, Approach 1 has a less influence on the enhancement of reliability, which suggests that different forms of expansion will contribute differently to the system reliability. By contrast, Approach 2 and 3 only add one road section, and the reliability increases remarkably. As a result, the expansion of the network should consider the actual traveling demand so as to improve the system reliability. 


\section{Conclusion}

In order to ease the traffic congestion, the exploration on reliability of road network were carried out. The connection reliability problem seems more prominent. Firstly, the reliability model of a complex road network with multiple OD pairs was established. Secondly, based on the Monte Carlo method and the breast-first search method, the connectivity reliability of the complex road network was calculated. Finally, the following conclusions were obtained:

(1) It is safer to use a single OD pair to define the road network reliability, whereas the presented model based on multiple OD pairs with different weighing coefficients is more practical to describe the actual road network system.

(2) The failure of nodes have a greater influences on the system reliability compared with that of the road sections.
For this reason, the node design is more important in the road network design and planning.

(3) The reinforcement of the damaged road sections and the expansion of the network will increase the connectivity reliability of the network. However, three approaches of expanding the network are compared, and the results show that the expansion of the road sections adjacent to the traveling points is a more effective and economical way to recover the reliability of road network system.

In this study, the analysis on road network connectivity reliability model is only applicable to the situation where road network system is damaged immediately by natural disasters or man-made deed.This is just a kind of the routine road network failure.Thus, more complex factors,such as the dynamic of road network, should be taken into consideration to modify the present road network model of connectivity reliability.

\section{References}

1. Qian YS, Wang M, Kang HX, Zeng JW, Liu YF. "Study on the road network connectivity reliability of valley city based on complex network", Mathematical Problems in Engineering, 2012, 2012, pp. 1-14

2. Qian Y S, Wang M, Kang H X, et al. "Study on the Road Network Connectivity Reliability of Valley City Based on Complex Network". Mathematical Problems in Engineering, 2012, 2012, pp. 1-12.

3. Chen A, Ji Z, Recker W. "Travel Time Reliability with RiskSensitive Travelers". Transportation Research Record, 20(1), 2002, pp. 177-179.

4. M A P T. "Dense network traffic models, travel time reliability and traffic management. II: Application to network reliability". Journal of Advanced Transportation, 33(2), 1999, pp.235-251.

5. M A P T. "Dense network traffic models, Travel time reliability and traffic management. I: General introduction". Journal of Advanced Transportation, 33(2), 1999, pp.218233.

6. Chen A, Kasikitwiwat P, Yang C. "Alternate capacity reliability measures for transportation networks". Journal of Advanced Transportation, 47(1), 2013, pp. 79-104.

7. Kuang A, Tang Z, Shan L. "Road Network Capacity Reliability Considering Travel Time Reliability". Procedia Social and Behavioral Sciences, 96(9), 2013, pp.1818-1827.

8. Tang T Q, Shi W F, Yang X B, et al. "A macro traffic flow model accounting for road capacity and reliability analysis". Physical A Statistical Mechanics \& Its Applications, 392(24), 2013, pp. 6300-6306.

9. Hou L W, Jiang F. "Simulation of Urban Road Network Reliability". Acta Simulata Systematica Sinica, 14(5), 2002, pp. 664-668.

10. NG Zhi hua YAO Zhi sheng SHAO Chun fu, Prof. "Travel Time Reliability in Road Network Associated with Road Section". China Safety Science Journal, 14(10), 2004, pp.8184.

11. Liang Y, Chen Y Y, Liu J F, et al. "The Application of Reliability in the Construction Investment Optimization of Road Network". Journal of Highway \& Transportation Research \& Development, 4(3), 2004, pp.4-11.

12. Leng J, Zhang Y, Zhao X, et al. "Travel Time Reliability of Urban Road Network Based on Generalized Travel Cost". Journal of Highway \& Transportation Research \& Development, 3(4), 2010, pp. 45-50.
13. Lu L M, Ms L W, Ms X Y, et al. "Zarit Caregiver Burden Interview: Development, reliability and validity of the Chinese version". Psychiatry \& Clinical Neurosciences, 63(6), 2009, pp. 730-734.

14. Leng J Q A, Zhang Y P B, Zhao Y P A, et al. "Assessment of Road Network Capacity Reliability Based on the Constraints of Link LOS". Journal of Transportation Systems Engineering \& Information Technology, 9(5), 2009, pp. 148-152.

15. Zhang J, Baiyan LI. "Research of image segmentation based on graph theory and minimum cut set algorithm". Laser Technology, 43(8), 2014, pp. 34-40.

16. 18. Cui T, Ma Y. "Research for connectivity reliability of directed acyclic network considering nodes and lines". Application Research of Computers, 45(31), 2015, pp. 43-53.

17. ZHU Shun-ying, W ANG Wei, DENG Wei, T ANG Yong, WAN G Bo. "Research on traffic network reliability and access road algorithm". China Journal of Highway and Transpiration, 13(1), 2000, pp.90-94.

18. Stephen C, David W. "Modeling network travel time reliability under stochastic demand". Transportation Research Part B: Methodological, 39 (2), 2005, pp. 119-140.

19. Zhang L, Shi Q, Dong S. "A Method of Road Network Optimization Based on Reliability Analysis with its Application", The Emerging Frontiers of Transportation and Development in China, 4(8), 2009, pp.3884-3892.

20. Erik J. "Network structure and travel patterns: explaining the geographical disparities of road network vulnerability". Journal of Transport Geography, 17 (3), 2009, pp. 234-244.

21. Kenetsu, U. "Estimating the value of travel time and of travel time reliability in road networks". Transportation Research Part B: Methodological, 66(9), 2014, pp. 129-147.

22. Chong P, Shuai B, Deng S, et al. "Analysis on Topological Properties of Dalian Hazardous Materials Road Transportation Network". Mathematical Problems in Engineering, 2015, 2015, pp.1-11.

23. Tozzo C, D'Andrea A, Cozzani D, et al. "Fatigue Investigation of the Interface Shear Performance in Asphalt Pavement". Modern Applied Science, 8(2), 2014, pp. 44-49.

24. Nakayama S I. "A study of perspective of road transportation reliability on the network level and investigation". Infrastructure Planning Review, 67(2), 2011, pp. 147-166. 